ISSN: 2316-6517

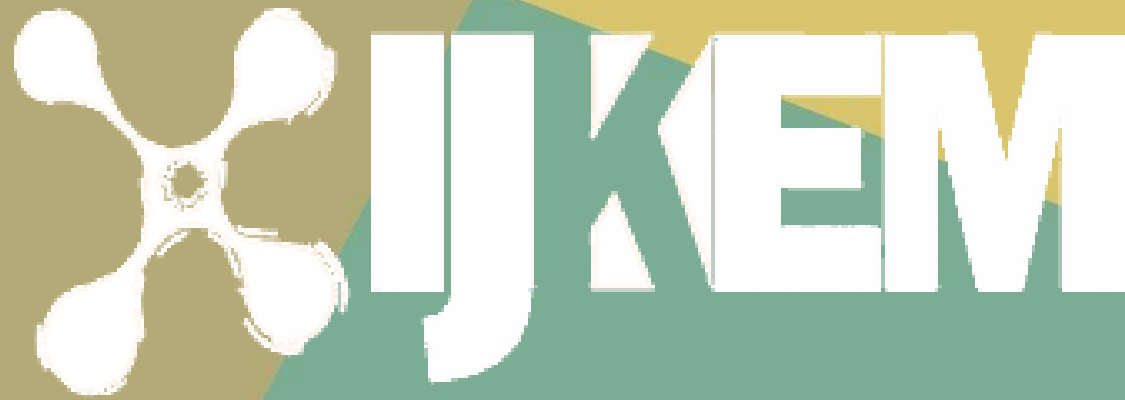

International Journal of Knowledge Engineering and Management

v. 9, n. $25,2020$.

(c) (1) (8)

ijkem.ufsc.br 
Florianópolis, v. 9, n. 25, p. 31- 52, 2020.

- ISSN 2316-6517 •

- DOI: 1047916 •

\section{PRODUÇÃO ACADÊMICA SOBRE INOVAÇÃO FRUGAL: UMA ANÁLISE BIBLIOMÉTRICA EM PERIÓDICOS INTERNACIONAIS NA ÚLTIMA DÉCADA}

\section{DENISE ADRIANA JOHANN}

Doutoranda em Administração

Universidade Federal de Santa Maria (UFSM)

johanndenisee@gmail.com

ORCID: 0000-0001-5769-3213

LUIS FELIPE DIAS LOPES

Doutor em Engenharia de Produção e Sistemas Universidade Federal de Santa Catarina (UFSC)

Iflopes67@yahoo.com.br

ORCID: 0000-0002-2438-0226

SIRLENE APARECIDA TAKEDA BRESCIANI

Doutoranda em Administração Universidade Estadual do Mato Grosso (UNEMAT)

sir takeda@hotmail.com ORCID: 0000-0003-0136-137X CLAUDETE CORREA DOS SANTOS Mestre em Administração Universidade do Vale do Itajaí (UNIVALI) claudetesantosadm@gmail.com ORCID: 0000-0003-2498-0384 VALÉRIA WISNIEWSKI PADILHA Doutora em Administração Universidade Estadual do Centro-Oeste (UNIVALI) vwpadilha@unicentro.br ORCID: 0000_0003-4120-4862

Submissão: 17/08/21 Aceitação: 01/09/21

Sistema de avaliação: duplo cego (double blind review). UNIVERSIDADE FEDERAL DE SANTA CATARINA (UFSC)

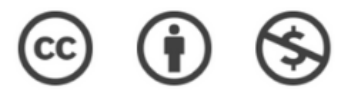


Florianópolis, v. 9, n. 25, p. 31- 52, 2020.

\section{PRODUÇÃO ACADÊMICA SOBRE INOVAÇÃO FRUGAL: UMA ANÁLISE BIBLIOMÉTRICA EM PERIÓDICOS INTERNACIONAIS NA ÚLTIMA DÉCADA}

\section{Resumo}

Objetivo: Analisar a produção científica na área de Inovação Frugal (IF), nas bases de dados Web of Science e Scopus (CAPES, 2021).

Metodologia: A pesquisa é caracterizada como descritiva com abordagem quantitativa e aplicação da técnica bibliométrica. Assim, após definir a técnica e tema, a busca foi realizada nas bases WoS e Scopus por tópico, utilizando os termos "frugal innovation", com intervalo de tempo de 2010 a 2020, resultando em 306 publicações na WoS e 396 na Scopus. Em seguida, os dados foram organizados na Planilha Excel e após, as publicações foram identificadas nas seguintes categorias: evolução temporal, áreas temáticas, publicações por países, autores e frequência de citações. Por fim, utilizouse o software VOSViewer para a criação de mapas textuais e clusters de co-citação.

Resultados: Destaca-se que a temática de Inovação Frugal vem crescendo em frequência de publicações, destacando-se o ano de 2020. Dentre os resultados, a Índia e Estados Unidos encontram-se entre os primeiros que mais publicam e em nona posição encontra-se o Brasil encontra-se em nona posição. Quanto a area, Gestão, Negócios e Contabilidade tem a primeira posição. Entre os anos de 201 a 2020, somando entre as bases, tem-se o total de 5.281 citações.

Originalidade/Valor: $\mathrm{O}$ uso da técnica de bibliometria apresenta-se como uma ferramenta relevante para a disseminação do conhecimento científico e tecnológico, possibilitando aos pesquisadores a análise quantitativa, descritiva e prognóstica de publicações realizadas em uma determinada área da ciência. Além disso, este estudo contribui com a disseminação do conhecimento sobre a inovação frugal, que é necessária para países que buscam o desenvolvimento econômico a partir da diminuição do uso de recursos e atendendo uma maior quantidade de pessoas, que antes não tinham acesso a determinados mercados e produtos.

Palavra-chave: Inovação, Inovação Frugal, Bibliometria. 


\section{International Journal of Knowledge Engineering and Management,}

Florianópolis, v. 9, n. 25, p. 31- 52, 2020.

- ISSN 2316-6517 •

- DOI: 1047916 •

\section{ACADEMIC PRODUCTION ON FRUGAL INNOVATION: A BIBLIOMETRIC ANALYSIS IN INTERNATIONAL JOURNALS IN THE LAST DECADE}

\section{Abstract}

Objective: This study deals with the scientific production of Frugal Innovation (IF) in the Web of Science and Scopus databases (CAPES, 2021). The choice of the area was due to the concern with the accelerated growth of the planet's population and the challenges found in all production chains, in this sense, offering high quality and low cost technology started to be studied by several economies, where frugal innovation can to be the central key in the future of innovation management, with the capacity to open a new path for solving problems, with socioeconomic results.

Methodology: The research is characterized as descriptive with a quantitative approach and application of the bibliometric technique. Thus, after defining the technique and theme, the search was carried out in the WoS and Scopus databases by topic, using the terms "frugal innovation", with a time interval from 2010 to 2020, resulting in 306 publications in WoS and 396 in Scopus. Then, the data were organized in an Excel spreadsheet and after, the publications were identified in the following categories: temporal evolution, thematic areas, publications by countries, authors and frequency of citations. Finally, the VOSViewer software was used to create textual maps and cocitation clusters.

Results: It is noteworthy that the topic of Frugal Innovation has been growing in frequency of publications, highlighting the year 2020. Among the results, India and the United States are among the first that publish the most and in ninth position is the Brazil is in ninth position. As for the area, Management, Business and Accounting has the first position. Between the years 2010 to 2020, adding between the bases, there is a total of 5,281 citations.

Originality/Value: The use of the bibliometric technique has been used as a relevant tool for the dissemination of scientific and technological knowledge, enabling researchers to carry out a quantitative, descriptive and prognostic analysis of publications carried out in a certain area of science. In addition, this study contributes to the dissemination of knowledge about frugal innovation, which is necessary for countries that seek economic development from the reduction in the use of resources and serving 
International Journal of Knowledge Engineering and Management,

Florianópolis, v. 9, n. 25, p. 31- 52, 2020.

-ISSN 2316-6517 •

- DOI: 1047916 •

a greater number of people, who previously did not have access to certain markets and products.

Keywords: Innovation, Frugal Innovation, Bibliometrics. 
International Journal of Knowledge Engineering and Management,

Florianópolis, v. 9, n. 25, p. 31- 52, 2020.

- ISSN 2316-6517 •

- DOI: 1047916 •

\section{Introdução}

O conhecimento tem sido força motriz desde os primórdios da evolução do homem à civilização. Desta forma, é considerado propulsor da economia, além de acelerar o ritmo da inovação, que surge diante da exploração e renovação contínua de conhecimentos (Jiménez, Costa \& Valle, 2014; Moustaghfir, Schiuma, 2013; Torres, Pierozzi, Pereira \& Castro, 2011). Neste cenário, o conhecimento se tornou um valor e as universidades são reconhecidas por sua promoção, aliado ao progresso científico (Atkinson \& Blanpied, 2008).

Sendo assim, é reconhecido que a maior parte do conhecimento é produzido nas universidades, sendo que esta instituição ocupa lugar central no mundo do conhecimento. Seu perfil decorre da expectativa da sociedade de que esta desempenhe seu papel no desenvolvimento e crescimento regional, sustentando a competitividade da economia (Leydesdorff \& Etzkowitz, 1998; Oliveira \& Moraes, 2016).

Dadas constantes e dinâmicas transformações, a sociedade tem se tornado dependente cada vez mais da capacidade de capital intelectual para o crescimento e desenvolvimento econômico e social (Quandt, Bezerra \& Ferraresi, 2015). Aliado a esses fatores, o crescimento acelerado da população do planeta desperta preocupação e alerta aos desafios encontrados em todas as cadeias de produção. Diante disso, oferecer tecnologia de alta qualidade e custo baixo, levando a disrupção em setores, passou a ser estudado por diversas economias, uma vez que a tecnologia pode reduzir os custos (Kunamaneni, 2019; Rao, 2017).

Prabhu (2017) afirma que a economia global enfrentará grandes desafios nas próximas décadas em termos de atender às necessidades de mais de sete bilhões de pessoas, ressaltando ainda, a prioridade em atender as necessidades básicas atuais não satisfeitas nos países pobres e em desenvolvimento. Dessa maneira, atingir esse crescimento com recursos disponíveis pode ser um dos desafios para as economias emergentes.

Diversos estudos na literatura relatam casos de empreendedorismo e inovação, considerados agentes de crescimento e desenvolvimento, por sua capacidade de fomentar mudanças econômicas e gerar emprego e renda (Baptista, Escária \& Madruga, 2008); (Drucker, 2016); (Schumpeter, 1982). 
International Journal of Knowledge Engineering and Management,

Florianópolis, v. 9, n. 25, p. 31- 52, 2020.

-ISSN 2316-6517 •

- DOI: 1047916 •

$\mathrm{Na}$ visão schumpeteriana a inovação é sugerida como uma atividade empreendedora que realiza novas combinações, onde indivíduos inovadores podem trazer mudanças sustentáveis, através da destruição criativa de produtos, serviços e processo para determinados mercados, onde a exploração de uma ideia ou invenção é denominada inovação (Elia, Margherita \& Petti, 2016; Schumpeter, 1934).

Para Crossan e Apaydin (2010), inovação é produção, assimilação e exploração de uma novidade de valor agregado nas esferas econômica e social através do desenvolvimento de novos métodos de produção e criação de novos sistemas de gestão. Dessa maneira, inovação é um processo, mas também um resultado.

Entre os diferentes tipos de inovação, tem-se a inovação frugal, que conforme Khan (2016), será chave central no futuro da gestão da inovação, com capacidade de proporcionar um novo caminho para as empresas. O journal The Economist (2010) está entre os primeiros divulgadores da temática, onde apresenta o conceito e ao mesmo tempo, argumenta que a inovação frugal veio para "quebrar todas as regras".

Os autores Tiwari \& Herstatt (2012) pontuam que todas as soluções frugais são caracterizadas pela acessibilidade, robustez, facilidade de uso, escalabilidade adicionadas a uma proposta de valor competitiva.

Diante disso, tem havido um aumento no interesse pela inovação frugal, o conceito de fazer mais com menos permitiu que mercados emergentes acessassem esses segmentos de mercado inexplorados (Prahalad \& Hart, 2008.). Essa combinação de recursos remete a definição de inovação de Schumpeter (1934) embora a ênfase aqui esteja nos recursos "disponíveis", e não naqueles que são "necessários" para uma inovação.

Diante do exposto, a busca por publicações pode contribuir para a exposição do estado da arte dessa temática. Assim, tem-se a seguinte questão de pesquisa: como se apresenta o cenário de publicações sobre inovação frugal? Nesse sentido, buscando responder ao questionamento, o objetivo desse estudo é analisar a produção científica na temática de Inovação Frugal (IF), nas bases de dados Web of Science e Scopus (CAPES, 2021). Para alcançar o objetivo na coleta de dados foi utilizada a técnica bibliométrica, nos períodos de 2010 a 2020.

\section{Inovação Frugal}




\section{International Journal of Knowledge Engineering and Management,}

Florianópolis, v. 9, n. 25, p. 31- 52, 2020.

- ISSN 2316-6517 •

- DOI: 1047916 •

A inovação frugal remete voltar ao básico, ao simples. Assim, autores comungam do pensamento da frugalidade levar ao desenvolvimento de produtos com características inovadoras e disruptivas, com a utilização de tecnologias e materiais já existentes, e que sejam capazes de atender um grupo novo de consumidores (Zeschky, Widenmayer \& Gassmann, 2011).

A criação de inovações frugais para Soni e Krishnan, (2014), significam uma inovação baseada na economia de escala focada nos recursos e na inclusão de parte da população até o momento desatendida, criando dessa forma, não somente valor econômico, mas também valor social. Dessa forma, as inovações frugais ganharam popularidade, partindo de mercados emergentes para economias desenvolvidas e principalmente em mercados que se encontram em processos de recuperação econômica (Zeschky, Widenmayer \& Gassmann, 2011).

Bhatti (2012) pontua que a IF se caracteriza pelas modificações sustentáveis e criação de valor para demandas e mercados com restrições de acesso. Hossain, Simula e Halme (2016) consideram a IF um modelo de negócio voltado a melhoria de produtos, serviços ou processos com recurso financeiro e tecnológico limitado. Corroborando, Rao (2013) argumenta que a IF pratica a reutilização de elementos para o desenvolvimento de um simples produto ou serviço, o que é mais aceito em países como a Índia e China devido as condições vividas por aquela população.

Sob essa perspectiva Radjou e Prabhu (2014) alertam à uma tendência emergente em torno da inovação frugal, a qual, cita que as empresas estão entendendo a urgência de serem frugais e inclusivas para ter sucesso na economia volátil do momento. Nesse sentido, observadores econômicos e políticos também estão refletindo sobre como formalizar outra forma de crescimento com dimensões sustentáveis, para a economia volátil de hoje, com o enfrentamento do rápido esgotamento de recursos naturais (Simula, Hossain \& Halme, 2015).

Weyrauch e Herstatt (2017) definiram três atributos de uma inovação frugal, sendo eles: I) reduzir de forma significativa os custos; II) concentrar as funcionalidades fundamentais e; III) aperfeiçoar o desempenho. Diante disso, esses critérios aliados ao fato do foco principal ser orientado ao usuário, considera-se que a IF pode ser avaliada no contexto de sustentabilidade social (Tiwari \& Herstat, 2012).

Apresentados os conceitos que definem a inovação frugal, na próxima sessão passa-se para a apresentação dos procedimentos metodológicos para a realização da 


\section{International Journal of Knowledge Engineering and Management,}

Florianópolis, v. 9, n. 25, p. 31- 52, 2020.

- ISSN 2316-6517 •

- DOI: 1047916 •

pesquisa bibliométrica, que buscou as publicações sobre a temática na plataforma Capes (2021).

\section{Procedimentos metodológicos}

A presente pesquisa é caracterizada como descritiva com abordagem quantitativa e aplicação da técnica bibliométrica. Desta maneira, Oliveira e Moraes (2016) explicam que a pesquisa bibliométrica se caracteriza por medir a influência dos periódicos e pesquisadores, com capacidade de evidenciar o perfil e tendência dos mesmos.

A realização da pesquisa dividiu-se em três etapas. Inicialmente foram utilizados os termos "frugal innovation" no campo de pesquisa da Web of Science e Scopus, com buscas no período de 2010 a 2020. Dessa forma, após coletar os dados na base, fezse a análise e apresentação a partir de gráficos e tabelas, das seguintes categorias: evolução temporal, áreas temáticas, publicações por países, autores e frequência de citações. Na quarta etapa, realizou-se a classificação das publicações e por fim foi realizada uma análise por meio de mapa textual gerado a partir do Software VOSViewer.

\section{Características Gerais das Publicações de Inovação Frugal na Web of}

\section{Science e Scopus}

Procurou-se ampliar o conhecimento referente às publicações relacionadas aos temas pesquisados, primeiramente digitaram-se as palavras "frugal innovation" como tópico no campo de pesquisa da Web of Science, delimitando-se o período de 2010 a 2020. Foram encontradas 306 publicações na base Web of Science e 396 publicações na base Scopus no período pesquisado.

Foi realizado levantamento de dados sobre evolução temporal, áreas temáticas, publicações por países, autores e frequência de citações. Assim, a Figura 1 demonstra a evolução temporal das publicações realizadas nas duas bases. 


\section{International Journal of Knowledge Engineering and Management,}

Florianópolis, v. 9, n. 25, p. 31- 52, 2020.

- ISSN 2316-6517 •

- DOI: 1047916 •

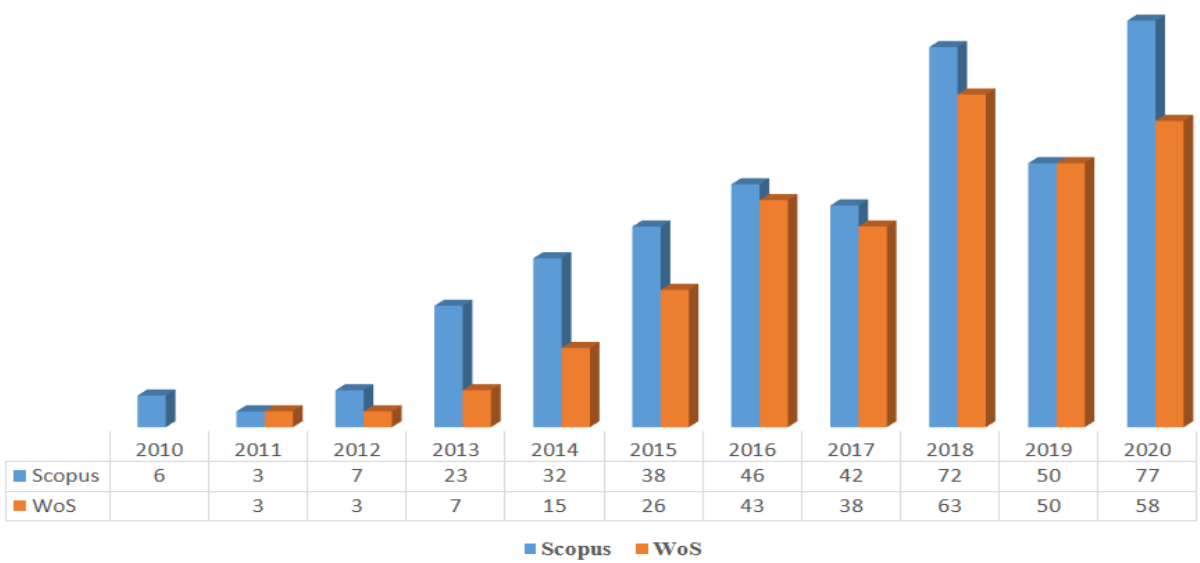

Figura 1 - Evolução temporal sobre Inovação Frugal. Fonte: WoS e Scopus (CAPES, 2021).

Sobre a evolução de publicações sobre IF, nota-se que a base Scopus apresenta maior quantidade durante o período pesquisado, sendo 396, enquanto na WoS foram encontradas 306 publicações. Considerando a última década, ressalta-se que em 2010 ainda não haviam publicações na base de dados WoS, enquanto na Scopus, havia um pequeno número de publicações. Na Scopus e na WoS no ano de 2018 ocorreu um elevado número de publicações nas respectivas bases, seguido por uma queda nas publicações em ambas as bases no ano de 2019. Já em 2020 as publicações retomam e a base Scopus se destaca com 77 publicações.

A Figura 2, apresenta o índice de áreas que mais publicam sobre a temática abordada neste trabalho. 


\section{International Journal of Knowledge Engineering and Management,}

Florianópolis, v. 9, n. 25, p. 31- 52, 2020.

- ISSN 2316-6517 •

- DOI: 1047916 •

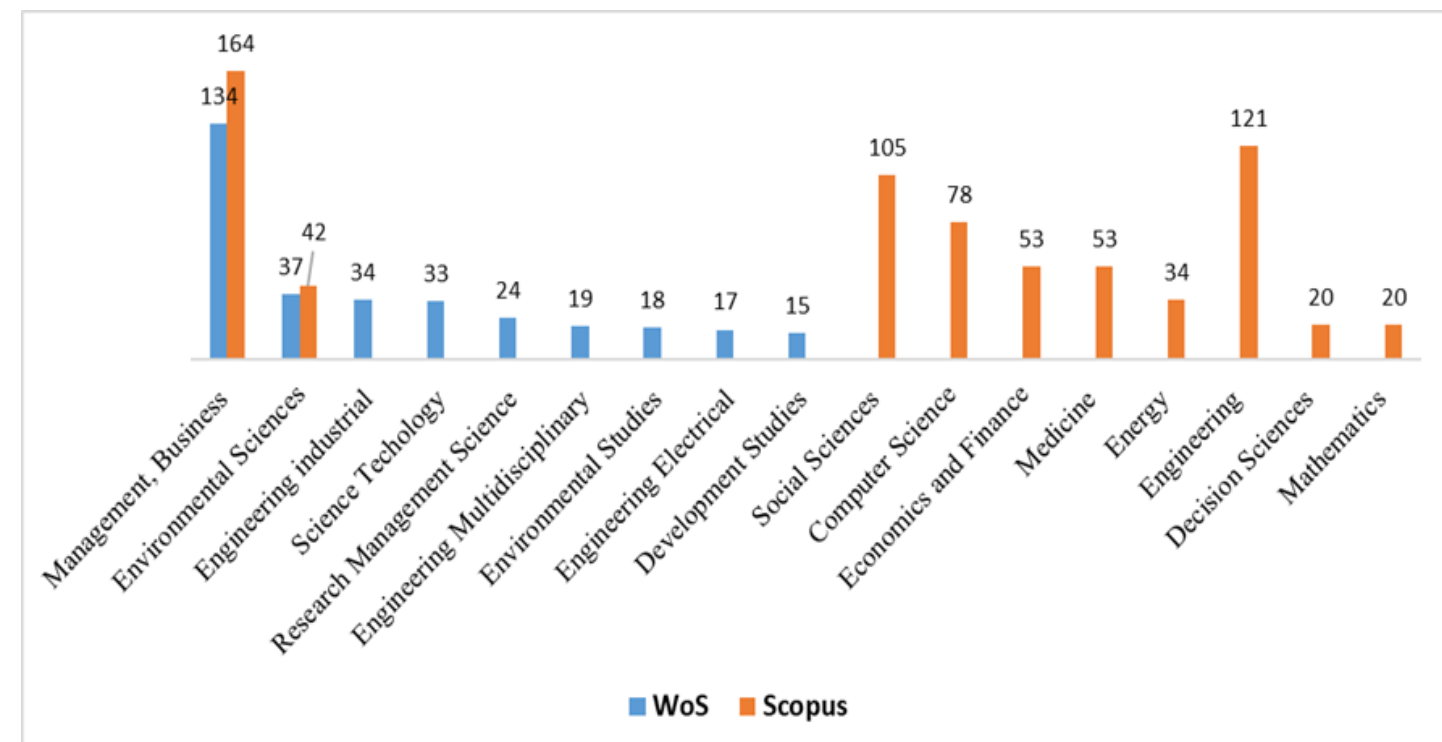

Figura 2 - Áreas de publicação sobre Inovação Frugal. Fonte: WoS e Scopus (CAPES, 2021).

A figura 2 mostra que as publicações são realizadas em diferentes áreas do conhecimento. Contudo, observa-se que as publicações apresentam familiaridade na área de Gestão, Negócios e Contabilidade, sendo 164 publicações na base Scopus. Já a WoS a área de gestão apresenta 134 publicações na área de negócios. Percebe-se que a base Scopus contém publicações direcionadas à área de saúde e, a base WoS em contrapartida, apresenta estudos voltados às engenharias.

A Figura 3, demonstra a distribuição de publicações conforme os países de origem. 


\section{International Journal of Knowledge Engineering and Management,}

Florianópolis, v. 9, n. 25, p. 31- 52, 2020.

- ISSN 2316-6517 •

- DOI: 1047916 •

Paises Publicações Inovaçõo

Frugal

Estados Unidos - 113

Alemarha-91

hdia- 115

United Kingdom/Inglaterra .

91

França- 76

Netheriands - 41

Finlàndia - 36

China -30

Brosi - 13

Bélgica - 25

Hóia-14

Figura 3 - Mapa de distribuição das publicações da temática Inovação Frugal no mundo. Fonte: autores a partir do uso do software mapchart.net.

Quanto ao número de publicações por países, tem-se a Índia com 115 publicações somadas de ambas as bases. Em segundo, o Estados Unidos com o total de 113 publicações, seguido pela Alemanha e Inglaterra, com 91 publicações. Em quarta posição tem-se a França com 76 publicações. Já o Brasil, encontra-se na nona posição, com o total de 13 publicações.

Tabela 1 - Índice de autores sobre Inovação Frugal

\begin{tabular}{l|c|c||c|c}
\hline Posição & Autores WoS & Quantidade & $\begin{array}{c}\text { Autores } \\
\text { Scopus }\end{array}$ & Quantidade \\
\hline $1^{\underline{a}}$ & Hossain, M & 8 & Bream, A. & 10 \\
$2^{\underline{a}}$ & Belkadi, F. & 7 & Maussion, P. & 9 \\
$3^{\underline{a}}$ & Bernard, A. & 7 & Hossain, M. & 7 \\
$4^{\underline{a}}$ & Borini. F. M. & 7 & Mourtzis, D. & 7 \\
$5^{\underline{a}}$ & Maussion, P. & 7 & Belkadi, F. & 6 \\
\hline
\end{tabular}

Fonte: WoS e Scopus (CAPES, 2021). 


\section{International Journal of Knowledge Engineering and Management,}

Florianópolis, v. 9, n. 25, p. 31- 52, 2020.

- ISSN 2316-6517 •

- DOI: 1047916 •

$\mathrm{Na}$ base Scopus, na primeira posição entre os dez autores que mais publicam sobre IF tem-se o autor Bream Alexander que atua na Universidade Erlargen Nuremberger, Alemanha. Na primeira posição da base de dados WoS e na terceira posição na Scopus aparece o autor Hossain M., que atua no departamento de Engenharia Industrial e Gestão na Aalto University, Espoo, Finlândia.

\subsection{Relatório de Citações}

Conforme Araújo (2006) a análise de citações é a categoria mais importante nos estudos com técnica bibliométrica, pois com os dados referentes às citações, descobrem-se itens relevantes ao estudo. O relatório de citações nos informa o $h$ (o número de estudos publicados e a frequência com que esses são citados). Na Figura 4 consta o número de citações por ano.

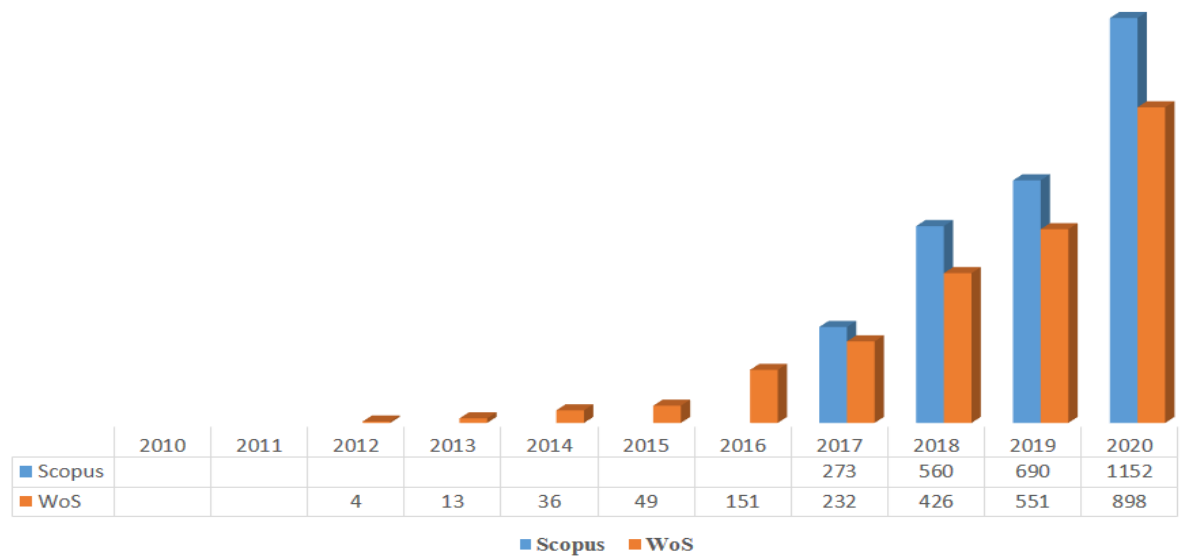

Figura 4 - Total de citações por ano. Fonte: WoS e Scopus (CAPES, 2021).

O período apurado totalizou 5.281 citações, sendo 2.506 citações na base $W e b$ of Science e 2.775 citações na Scopus. Nesse sentido, pode-se perceber que coincidindo com a ausência de estudos publicados nas bases, durante o período de 2010 a 2011 não houve citações, sendo assim, somente a partir de 2012 ocorrem as primeiras citações na base de dados WoS e no ano de 2017 as primeiras citações na base de dados Scopus (273 citações). A partir do ano de 2018, as citações crescem de forma exponencial. 


\section{International Journal of Knowledge Engineering and Management,}

Florianópolis, v. 9, n. 25, p. 31- 52, 2020.

- ISSN 2316-6517 •

- DOI: 1047916 •

Seguindo, por meio da análise de citações, identificaram-se as publicações mais citados sobre inovação frugal nas respectivas bases de dados. Desta maneira, o Quadro 1 apresenta os títulos mais citados na base de dados Web of Science.

Quadro 1 - Estudos com maior frequência de citações na base de dados WoS

\begin{tabular}{|l|c|c|}
\hline \multicolumn{1}{|c|}{ Referência } & Ano & $\begin{array}{c}\text { Freq. } \\
\text { Citações }\end{array}$ \\
\hline $\begin{array}{l}\text { Frugal innovation in emerging markets: the case of Mettler Toledo. Por: } \\
\text { Zeschky, M., Widnmayer, B. \& Gassmann, O. Ressarch-Tecnology } \\
\text { Management, v. 54, i. 4, p. 38-45 }\end{array}$ & 2011 & 175 \\
\hline $\begin{array}{l}\text { Towards a sufficiency-driven business model: experiences and } \\
\text { opportunities. Por: Bocken, N. M. P. \& Short, S. W. Enviromental } \\
\text { Innovation and Societal Transitions. v. 18, p. 41-61 }\end{array}$ & 2016 & 112 \\
\hline $\begin{array}{l}\text { From cost to frugal and reverse innovation: mapping the field and } \\
\text { implications for global competitiveness. Por: Zeschky, M. Winterhalter, S. } \\
\text { \& Gassmann, O. Research Technology Management. v. 57, i. 4, p. 20- } \\
27\end{array}$ & 2014 & 97 \\
\hline $\begin{array}{l}\text { Resource-constrained product development: implications for green } \\
\text { marketin and green supply chains. Por: Sharma, A. \& lyer, G. Industrial } \\
\text { Marketing Management. v. 41, i. 4, p. 599-608 }\end{array}$ & 2012 & 89 \\
\hline $\begin{array}{l}\text { Product innovation for the people's car in na emerging economy. Por: } \\
\text { Ray, S. \& Ray, P. K. Technovation. v. 31, i. 5-6, p. 216-227 }\end{array}$ & 2011 & 81 \\
\hline
\end{tabular}

Fonte: Web of Science (CAPES, 2021).

Os autores Zeschky, Widnmayer e Gassman (2011) em seu estudo intitulado Inovação frugal em mercados emergentes: o caso da Mettler Toledo, trazem exemplos práticos de aplicações de inovação frugal, apresentando o veículo Tatá Nano desenvolvido pela Tatá Motors na Índia; a máquina de ultra-som portátil desenvolvida pela General Eletric - GE na China, entre outros. Após, pontuam sobre os desafios da inovação em mercados emergentes ocidentais, relacionando ao rápido crescimento da quantidade de pessoas que tem acessado a classe média, que mesmo tendo poder de compra, apresentam restrições de renda. A metodologia do estudo foi de natureza qualitativa com estudo de caso na R\&D, subsidiária chinesa da Western MNCs, sendo: balança Mettler Toledo Basic; máquina de ultra-som portátil GE; mouse M215 Logitch; sistema de monitoramento de pacientes Philips e; tomografia computadorizada Siemens.

As análises apresentam as características dos produtos, motivação para 0 desenvolvimento dos produtos e, desenvolvimento e implementação dos produtos. Nas considerações finais recomendam que seja dada atenção à promoção da mentalidade frugal nas equipes de P\&D, capacitando-as e, ao mesmo tempo, essas organizações 


\section{International Journal of Knowledge Engineering and Management,}

Florianópolis, v. 9, n. 25, p. 31- 52, 2020.

- ISSN 2316-6517 •

- DOI: 1047916 •

necessitam criar em suas subsidiarias, uma estrutura capaz de atender as necessidades locais. No Quadro 2, tem-se os cinco estudos mais citados na base de dados Scopus.

Quadro 2 - Estudos com maior frequência de citações na base de dados Scopus

\begin{tabular}{|l|c|c|}
\hline \multicolumn{1}{|c|}{ Referência } & Ano & $\begin{array}{c}\text { Freq. } \\
\text { Citações }\end{array}$ \\
\hline $\begin{array}{l}\text { Towards a sufficiency-driven business model: experiences and } \\
\text { opportunities. Por: Bocken, N. M. P. \& Short, S. W. Enviromental } \\
\text { Innovation and Societal Transitions. v. 18, p. 41-61 }\end{array}$ & 2016 & 125 \\
\hline $\begin{array}{l}\text { From cost to frugal and reverse innovation: mapping the field and } \\
\text { implications for global competitiveness. Por: Zeschky, M. Winterhalter, S. } \\
\text { \& Gassmann, O. Research Technology Management. v. 57, i. 4, p. 20- } \\
27\end{array}$ & 2014 & 125 \\
\hline $\begin{array}{l}\text { Resource-constrained product development: implications for green } \\
\text { marketin and green supply chains. Por: Sharma, A. \& lyer, G. Industrial } \\
\text { Marketing Management. v. 41, i. 4, p. 599-608 }\end{array}$ & 2012 & 95 \\
\hline $\begin{array}{l}\text { Product innovation for the people's car in na emerging economy. Por: } \\
\text { Ray, S. \& Ray, P. K. Technovation. v. 31, i. 5-6, p. 216-227 }\end{array}$ & 2011 & 90 \\
\hline $\begin{array}{l}\text { How disruptive is frugal? Por: Rao, B. C. Technology in Society, v. 35, i. } \\
\text { 1, p. 65-73 }\end{array}$ & 2013 & 86 \\
\hline
\end{tabular}

Fonte: Scopus (CAPES, 2021).

Comparando os estudos com maior frequência de citações nas duas bases, pode-se observar que os quatro destes encontram-se em ambas as bases, mas em posições de destaque diferentes. Ainda, enquanto no Quadro 1 tem-se duas publicações dos autores Zeschky e Gassmann, neste, o autor Rao, antes não destacado, alcança a quinta posição nas frequências de citações.

Conforme os dados apresentados no Quadro 2, na base Scopus, os autores Bocken e Short se destacam em primeiro lugar com o estudo intitulado "Rumo a um modelo de negócios orientado à suficiência: experiências e oportunidades". Neste, iniciam com o apontamento sobre a necessidade das organizações se capacitarem para o desafio de pensar de forma disruptiva, com o propósito de projetar e difundir inovações voltadas para as necessidades locais. O objetivo geral do estudo foi entender como as escolhas da Tatá Motors em relação ao uso de tecnologia, design de produto e práticas organizacionais para o desenvolvimento de novos produtos, permitiram enfrentar 0 desafio de inovação para o mercado de baixa renda da Índia.

Apresentados os resultados de frequência de citações, passa-se para o mapa de co-citações de autores. Dessa forma, a análise de co-citação permite analisar os clusters existentes e os autores que pertencem a eles, bem como prospectar grupos ou 


\section{International Journal of Knowledge Engineering and Management,}

Florianópolis, v. 9, n. 25, p. 31- 52, 2020.

- ISSN 2316-6517 •

- DOI: 1047916 •

pares de autores citados em conjunto em um mesmo estudo e ainda identificar grupos de autores ou teorias em comum (Ramos-Rodríguez \& Ruiz-Navarro, 2004).

Foram selecionados autores citados pelo menos 20 vezes, verificando os possíveis grupos ou pares de publicações que são citadas em conjunto com outra publicação. Para isso, utilizou-se o software VOSViewer para identificar os possíveis clusters de autores e suas relações, bem como criação de mapa de relacionamentos e mapa de palavras chaves que aparecem com frequência no texto.

De acordo com Hair, Tatham, Anderson e Black (1998), os clusters formados possuem características em comum. A Figura 5 representa o Mapa de co-citação na base de dados Web of Science.

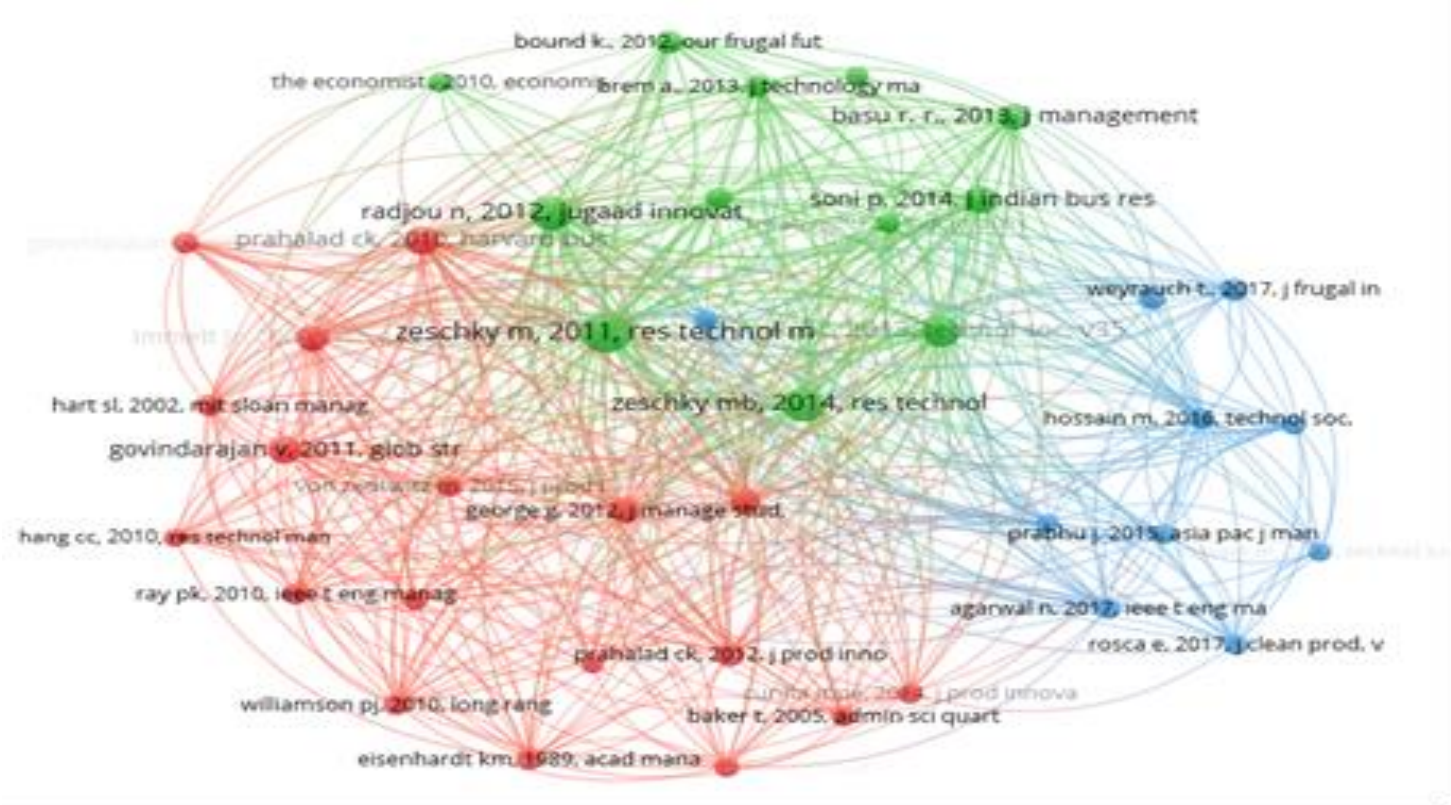

Figura 5 - Mapa de co-citação na base de dados WoS. Fonte: Elaborado pelos autores por meio do VOSViewer.

Das 25.239 referências citadas na base de dados Web of Science, 55 foram citados mais de 20 vezes. Como pode-se observar no Quadro 3, o autor Zeschky aparece com 94 citações em 2011 e 69 em 2014. o autor Rao aparece com 62 citações, seguido do autor Radjou com 72 citações, ambos aparecendo entre os 3 autores mais citados. O maior cluster é o vermelho, composto por 18 autores, entre eles, Zeschky, Govindarajan e Prahalad. O cluster de cor verde aparece com 12 autores, com destaque 


\section{International Journal of Knowledge Engineering and Management,}

Florianópolis, v. 9, n. 25, p. 31- 52, 2020.

-ISSN 2316-6517 •

- DOI: 1047916 •

para Radjou e Tiwari e por último, o cluster de azul com um total de 10 citações, entre elas, do autor Weyrauch com 31 citações.

Seguindo, as análises de palavras mais citadas nas publicações da WoS foram realizadas com o software VOSviewer, nos campos de título e resumo. Inicialmente, foram selecionados os termos com mais de 10 ocorrências. A partir de então, o software identificou um total de 204 palavras chaves, relevantes a temática abordada, as quais foram selecionadas e agrupadas em 6 clusters. Corroborando, Van Eck e Waltman (2010) citam que quanto mais importante um item, tanto maior será a sua escrita e o seu círculo representativo.

A Figura 6 apresenta 204 palavras chaves com mais de 10 ocorrências de um total de 7.987 links.

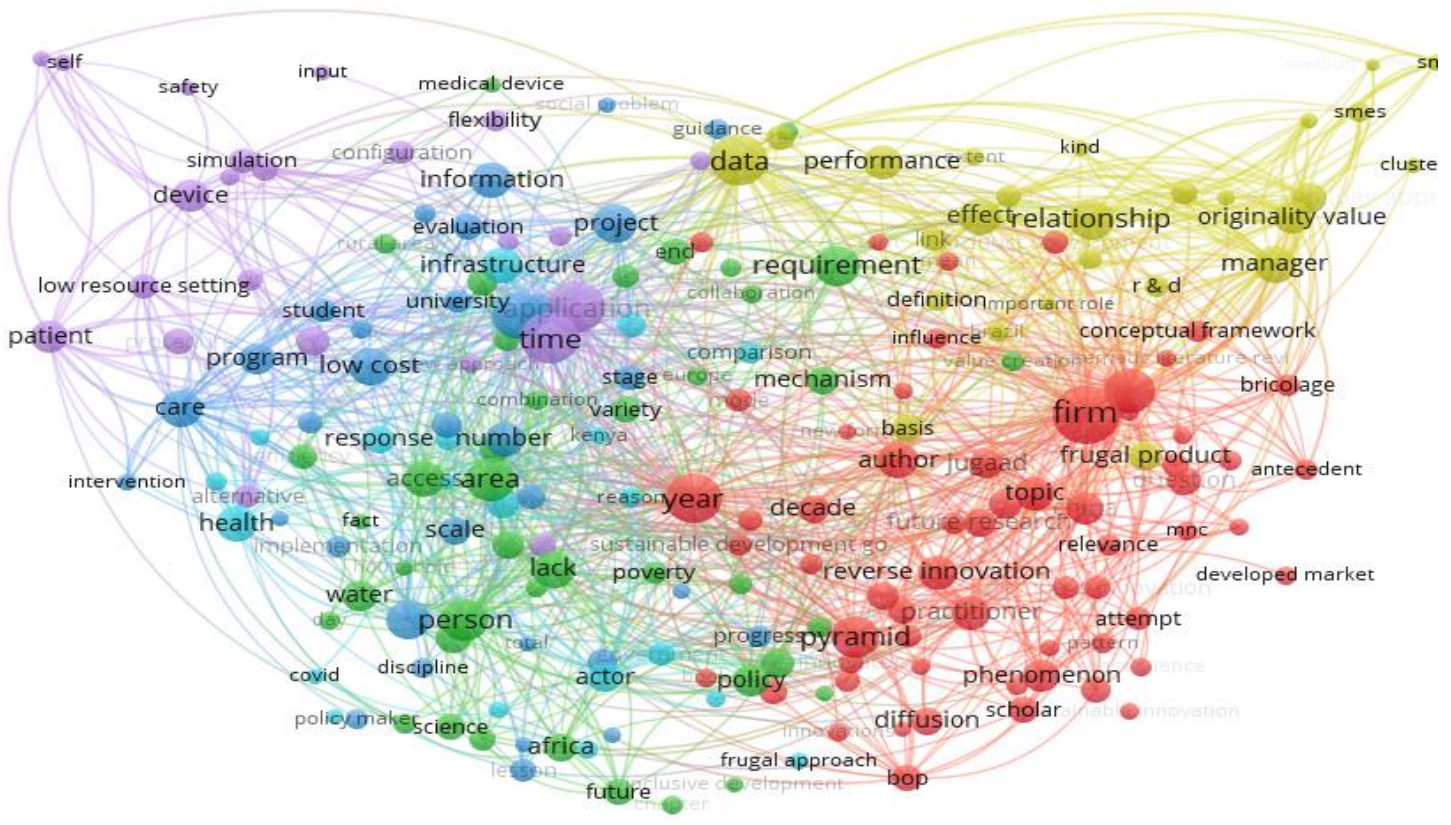

Figura 6 - Rede de palavras. Fonte: Elaborado pelos autores por meio do software

vosviewer.

Por meio da análise das palavras-chaves das 306 publicações encontrados na base de dados WoS e 396 de 10.327 palavras chaves, o total de 340 termos foram utilizados com maior frequência, ou seja, os termos selecionados para nosso estudo aparecem mais de 10 vezes. Diante disso, na rede verificam-se seis clusters predominantes, o principal corresponde às palavras elencadas no grupo firm em 


\section{International Journal of Knowledge Engineering and Management,}

Florianópolis, v. 9, n. 25, p. 31- 52, 2020.

- ISSN 2316-6517 •

- DOI: 1047916 •

vermelho com 61 itens, destacando palavras como bricolage, capability, frugal, innovation, process, jugaad, pyramid, sustainble innovation, entre outras.

O segundo cluster, de cor verde com 41 termos, tem-se em destaque termos como person, requeriment, policy, Africa, área, water e science. Já o terceiro cluster de cor azul escuro é formado por 33 itens como information, project, low cost, care e evaluation. Enquanto o quarto cluster, de cor amarela, formado por 26 palavras-chaves com destaque para Brazil e frugal product. O quinto cluster de cor roxa, é composto por 23 itens, com palavras-chaves como investment, limitation e flexibility. Por fim, o sexto cluster de cor azul clara, é composto por 20 palavras chaves, entre elas, government, covid e frugal approach.

\section{Considerações Finais}

Este estudo teve como objetivo analisar as publicações da temática de inovação frugal através de um estudo bibliométrico. Para isso, foram analisadas publicações das bases Web of Science e Scopus (CAPES, 2021). Assim, encontrou-se o total de 702 publicações no período analisado, apresentando crescente aumento, sendo a maior quantidade no ano de 2020 com o total de 135, enquanto em 2019 a quantidade foi de 100 publicações.

Entre os países que se destacam entre as publicações sobre IF, tem-se primeiramente a Índia e Estados Unidos, enquanto o Brasil encontra-se na nona posição em quantidade de publicações. As publicações são realizadas em diferentes áreas, sendo a maior quantidade na área de Gestão, Negócios e Contabilidade. Quanto a frequência de citações, de 2010 a 2020 foram feitas o total de 5.281 citações, sendo 2.506 citações na base Web of Science e 2.775 citações na Scopus.

Ainda, a partir do banco de dados, realizou-se análise de citação e co-citação de autores, bem como uma análise textual das palavras do resumo e palavras-chave dos trabalhos. A análise das publicações referente ao termo inovação frugal (innovation frugal) evidenciando que a IF apresenta forte envolvimento com as palavras bricolage, capability, frugal, innovation, process, jugaad, pyramid, sustainble innovation.

Assim, autores contribuem com o pensamento da frugalidade levar ao desenvolvimento de produtos, com características inovadoras e disruptivas, com a 


\section{International Journal of Knowledge Engineering and Management,}

Florianópolis, v. 9, n. 25, p. 31- 52, 2020.

- ISSN 2316-6517 •

- DOI: 1047916 •

utilização de tecnologias e materiais já existentes, e que sejam capazes de atender um grupo novo de consumidores, com baixo custo e qualidade.

Por fim, afirma-se que o estudo evidenciou que a temática vem crescendo em meio aos pesquisadores de diferentes áreas, em países com diferentes posicionamentos econômicos. Diante disso, enquanto agenda de pesquisa, sugere-se a busca por aprofundamento de estudos em torno dos laboratórios frugais em algumas universidades e também um aprofundamento maior em produtos frugais comercializados, para um entendimento maior de como está o avanço da Inovação Frugal de forma prática, ou seja, o desenvolvimento dos produtos, serviços e processos frugais e sua aceitação no mercado.

\section{Referências bibliográficas}

Baptista, R., Escaria, V.\& Madruga, P. (2008). Entrepreneurship, regional development and job creation: the case of Portugal. Small Business Economics, 30(1),49-58.

Bhatti, Y. A. (2012). What is Frugal, What is Innovation? Towards a Theory of Frugal Innovation. SSRN Electronic Journal, p. 1-45.

Bouchery, Y.; Ghaffari, A.; Jemai, Z. \& Fransoo, J. (2016). Sustainable transportationand order quantity: insights from multiobjective optimization. Flexible Services and Manufacturing Journal, 28 (3), 367-396.

Crossan, M. M.; Apaydin, M. (2010). A multi- dimensional framework of organizational innovation: A systematic review of the literature. Journal of Management Studies, 47 (6), 1154-1191.

Drucker, P. F. (2016). Inovação e Espírito Empreendedor: (entrepreneurship) : práticase princípios. tradução de: Carlos J. Malferrari. São Paulo: Cengage Learning.

Elia, G.; Margherita, A.\& Petti, C. (2016). An operational model to develop technology entrepreneurship EGO-system, International Journal of Innovation and Technology Management, 13 (5), 164-182. 


\section{International Journal of Knowledge Engineering and Management,}

Florianópolis, v. 9, n. 25, p. 31- 52, 2020.

- ISSN 2316-6517 •

- DOI: 1047916 •

Hair, J.F.; Tatham, R. L.; Anderson, R. E.\& Black, W. C. (1998). Análise multivariada de dados. Tradução Adonai Schlup Sant'Anna e Anselmo Chaves Neto. 5. ed. Porto Alegre: Bookman.

Hossain, M.; Simula, H.\& Halme, M. (2016) Can Frugal go global? Diffusion patterns of frugal innovations. Technology in Society, 46, 132-139.

Jiménez, D. J.; Costa, M. M. \& Valle, R. S. (2014). Knowledge management practices for innovation: a multinational corporation's perspective. Journal of Knowledge Management, 18 (5), 905-918.

Khan, R. (2016). How frugal innovation Promotes Social Sustainability. Sustainability, 8 (1034), 1-29.

Kunamaneni, S. (2019). Challenges in moving from incremental to radical low-cost innovation in emerging and transition countries: institutional perspectives based on rechargeable battery innovation in China and point-of-use water purification innovation in India. International Journal of Innovation Management, 23(03), 1-36.

Leydesdorff, L.\& Etzkowitz, H. (1998). The Triple Helix as a model for innovationstudies. Science and Public Policy, 25(3), 195-203.

Moustaghfir, K.\& Schiuma, G. Knowledge, learning, and innovation: research and perspectives. Journal of Knowledge Management, 17(4), 495 - 510.

Oliveira, J.\& Moraes, K. (2016). Produção do conhecimento na universidade pública no brasil: tensões, tendências e desafios. Educação em Revista. Belo Horizonte, 32(4).

Prabhu, J. (2017) Frugal innovation: doing more with less for more,

PhilosophicalTransactions of the Royal Society A, 375 (2095), 375.

Quandt, C. O.; Bezerra, C. A. \& Ferraresi, A. A. (2015). Dimensions of organizational innovativeness and its impact on innovation performance: proposition and evaluation of a model. Gestão \& Produção, 22(4), 873-886.

Prahalad, C. K.\& Hart, S. L. (2008). The fortune at the bottom of the pyramid. Estratégias e Negócios, Florianópolis, 1(2), 1-23.

Radjou, N. \& Prabhu, J. (2014). What frugal innovators do. Harvard Business Review, v. 10. 


\section{International Journal of Knowledge Engineering and Management,}

Florianópolis, v. 9, n. 25, p. 31- 52, 2020.

- ISSN 2316-6517 •

- DOI: 1047916 •

Ramos-Rodríguez, A. R \& Ruíz-Navarro, J. (2004). Changes in the intellectual structure of strategic management research: a bibliometric study of the Strategic Management Journal 1980-2000. Strategic Management Journal, 25, 981-1004.

Rao, B. C. (2017). Advances in science and technology through frugality. IEEE EngineeringManagement Review, 45 (1), 32-38.

Schumpeter, J. A. (1934). A Teoria do Desenvolvimento Econômico: Uma Investigação sobre Lucros, Capital, Crédito, Juros e o Ciclo de Negócios, Transaction Publishers, Cambridge.

Schumpeter, J. A. (1982). Teoria do desenvolvimento econômico: uma investigação sobre lucros, capital, crédito, juro e o ciclo econômico. Abril Cultural.

Simula, H.; Hossain, M. \& Halme, M. (2015). Frugal and reverse innovations - Quo Vadis? Current Science, 109 (5), 1567-1572.

Tiwari, R. \& Herstatt, C. (2012). India-a lead market for frugal innovations? Extending the lead market theory to emerging economies. TIM/TUHH Working Paper, n. 67.

Torres, T. Z.; Pierozzi, J. I.; Pereira, N. R. \& CASTRO, A. de. Knowledge management and communication in Brazilian agricultural research: An integrated procedural approach. International Journal of Information Management. v. 31, p.121-127.

Weyrauch, T. \& Herstatt, C. (2017). What is frugal innovation? Three defining criteria. Journal of Frugal Innovation, 2 (1).

Zeschky, M.; Widenmayer, B. \& Gassmann, O. (2011). Frugal Innovation in Emerging Markets. Research-Technology Management, 54(4), 38-45. 